\title{
Brownian motor efficiency enhanced by nonequilibrium noise
}

\author{
Jakub Spiechowicz \\ Institute of Physics \\ University of Silesia \\ 40-007 Katowice, Poland \\ Email: j.spiechowicz@gmail.com
}

\author{
Peter Hänggi \\ Institute of Physics \\ University of Augsburg \\ 86135 Augsburg, Germany \\ Email: hanggi@physik.uni-augsburg.de
}

\author{
Jerzy Łuczka \\ Institute of Physics \\ University of Silesia \\ 40-007 Katowice, Poland \\ Email: jerzy.luczka@us.edu.pl
}

\begin{abstract}
We study transport properties of an inertial Brownian motor which moves in a symmetric spatially periodic potential and is subjected to both a symmetric, unbiased time-periodic driving $a \cos (\omega t)$ and a static constant force $F$ or, likewise, nonequlibrium noise of equal mean value $\langle\eta(t)\rangle=F$. We focus on the efficiency of the motor and discuss various definitions. We show that within selected parameter regimes, nonequilibrium noise $\eta(t)$ can be significantly more effective than the deterministic force $F$. This implies that the motor moves much faster and its efficiency distinctly increases. These features are independent of the assumed Brownian motor efficiency measure. We demonstrate this feature with detailed simulations by resource to generalized white Poissonian noise. Our theoretical results can be tested and corroborated experimentally by use of a setup that consists of a resistively and capacitively shunted Josephson junction.
\end{abstract}

\section{INTRODUCTION}

Interaction of nonlinearity and noise in nonequilibrium systems often gives rise to subtle and counterintuitive phenomena, in the sense that those might seem at first glance to contradict our everyday experience. Prominent examples include stochastic resonance [1], Brownian ratchets [2], [3], molecular motors and machines [4], or intra-celluar transport [5], to name only a few. In particular, transport processes in the microscale domain in situ occur in strongly fluctuating environments. Typically randomness hinders directed transport manifested in quantifiers such as the average velocity of a Brownian particle. However, a constructive role of both equilibrium and nonequilibrium fluctuations has since been demonstrated in many noise-assisted phenomena including enhancement of diffusion [6] and amplification of transport efficiency [7]. The standard way to move particles into a desired direction is to apply a constant force $F$ pointing in this direction. Here we show that a stochastic force $\eta(t)$ of equal mean value as $F$ can be much more effective than its deterministic counterpart. We demonstrate this fact with an archetypal model of Brownian motor. In doing so, we concentrate on an unusual regime in which the Brownian particle moves in a direction opposite to a biasing force thus indicating the anomalous transport behaviour in the form of Absolute Negative Mobility (ANM) [8]-[13].

The layout of the present work is as follows. In Sec. II we describe a mathematical model of the inertial Brownian motor which is driven by both a time periodic and constant force $F$ or biased noise $\eta(t)$. In the next part we present the detailed analysis of three quantifiers characterizing efficiency of the Brownian motor. The last section provides a summary and the conclusions.

\section{FORMUALTION OF THE MODEL}

We consider a classical Brownian particle of mass $M=$ 1 moving in a one dimensional periodic potential $V(x)=$ $V(x+1)=\sin 2 \pi x$ and driven by an unbiased time-periodic force $a \cos (\omega t)$ with amplitude $a$ and angular frequency $\omega$. We study two scenarios: the system is subjected to (i) a constant force $F$ or (ii) nonequlibrium noise $\eta(t)$. In order to compare these two cases we set the mean value of noise $\eta(t)$ equal to $F$, namely $\langle\eta(t)\rangle=F$. The corresponding dimensionless Langevin equations read

$$
\begin{aligned}
& \ddot{x}+\gamma \dot{x}=-V^{\prime}(x)+a \cos (\omega t)+\sqrt{2 \gamma D_{T}} \xi(t)+F, \\
& \ddot{x}+\gamma \dot{x}=-V^{\prime}(x)+a \cos (\omega t)+\sqrt{2 \gamma D_{T}} \xi(t)+\eta(t),
\end{aligned}
$$

where the dot and the prime denote a differentiation with respect to time $t$ and position $x$ of the Brownian particle, respectively. The parameter $\gamma$ is the friction coefficient. Thermal equilibrium noise is modeled by symmetric and unbiased $\delta$ correlated Gaussian white noise $\xi(t)$ of mean $\langle\xi(t)\rangle=0$ and the correlation function $\langle\xi(t) \xi(s)\rangle=\delta(t-s)$. Its intensity $D_{T} \propto k_{B} T$ is proportional to the thermal energy, where $T$ is temperature and $k_{B}$ is the Boltzmann constant. The dimensional version of Eqs. (1) and corresponding scalings of time and the particle position is presented in Ref. [14]. From Eq. (1a) it follows that the average particle velocity $\langle v(F)\rangle$ obeys the relation $\langle v(F)\rangle=-\langle v(-F)\rangle$. In particular, $\langle v(0)\rangle=0$, so for $F=0$ there is no directed transport in the asymptotic long-time regime. The static force $F \neq 0$ breaks the symmetry and therefore induces a directed motion of the particle. From the above it follows that we can limit our considerations to the case of positive values of the force $F>0$.

As a model of biased noise $\eta(t)>0$ we choose generalized white Poissonian noise [15], reading

$$
\eta(t)=\sum_{i=1}^{n(t)} z_{i} \delta\left(t-t_{i}\right)
$$

where $t_{i}$ are the random times of a Poissonian counting process $n(t)$ with the parameter $\lambda$. This noise is a random sequence of $\delta$-pulses with random amplitudes $z_{i}$. The probability that $k$ pulses occur in the interval $(0, t)$ is given by the Poisson 
distribution $\operatorname{Pr}\{n(t)=k\}=(\lambda t)^{k} \exp (-\lambda t) / k$ !, with $\lambda$ being the mean number of $\delta$-pulses per unit time. The amplitudes $\left\{z_{i}\right\}$ are mutually independent random variables of a common density $\rho(z)$ and are independent of the counting process $n(t)$. Without loosing of generality, we can assume that they are exponentially distributed; i.e., $\rho(z)=\zeta^{-1} \theta(z) \exp (-z / \zeta)$, where $\theta(z)$ is the Heaviside step function. In consequence, all amplitudes $\left\{z_{i}\right\}$ are positive and realizations of the process $\eta(t)$ are non-negative, i.e. $\eta(t) \geq 0$. This biased nonequilibrium noise thus has finite mean $\langle\eta(t)\rangle=\lambda\left\langle z_{i}\right\rangle=\sqrt{\lambda D_{P}}$ and the covariance function $(\langle\eta(t) \eta(s)\rangle-\langle\eta(t)\rangle\langle\eta(s)\rangle)=$ $2 D_{P} \delta(t-s)$. We introduced the Poisson noise intensity $D_{P}=$ $\lambda\left\langle z_{i}^{2}\right\rangle / 2=\lambda \zeta^{2}$, where $\left\langle z_{i}^{k}\right\rangle=k ! \zeta^{k}$ are statistical moments of the amplitudes $\left\{z_{i}\right\}$. We also assume that thermal equilibrium fluctuations $\xi(t)$ are uncorrelated with nonequilibrium noise $\eta(t)$; i.e., $\langle\xi(t) \eta(s)\rangle=\langle\xi(t)\rangle\langle\eta(s)\rangle=0$. The influence of the Poissonian noise parameters $\lambda$ and $D_{P}$ on stochastic realizations of $\eta(t)$ is presented in [14]. Here, we only mention two extreme regimes. The first limiting case is for both, $\lambda$ and $D_{P}$ large, implying that the particle becomes frequently kicked by large $\delta$-pulses. On the contrary, when both $\lambda$ and $D_{P}$ are small, then the particle is only rarely kicked by $\delta$-pulses of small amplitudes.

\section{BROWNIAN MOTOR EFFICIENCY}

The crucial transport quantity characterizing a Brownian motor is its average velocity. In the asymptotic long time regime, it is determined by the relation [16]

$$
\langle v\rangle=\lim _{t \rightarrow \infty} \frac{\omega}{2 \pi} \int_{t}^{t+2 \pi / \omega} \mathbb{E}[v(s)] d s,
$$

where $\mathbb{E}[v(t)]$ denotes the average of the actual velocity $v(t)=$ $\dot{x}(t)$ over the noise realizations and initial conditions. However, it is not necessarily of a decisive character in attaining optimal efficiency for the working operation. For example, a large transport velocity is of little use if the fluctuations are too erratic around the average velocity, thus spoiling effectiveness. We next study the magnitude of the velocity fluctuations. In the long time regime these are given by the variance

$$
\sigma_{v}^{2}=\left\langle v^{2}\right\rangle-\langle v\rangle^{2}
$$

If the variance is large, i.e. if $\left.\sigma_{v}\right\rangle\langle v\rangle$, the Brownian motor can move for some time in the direction opposite to its average velocity $\langle v\rangle$.

As a measure of the transport effectiveness, we use efficiency defined as the ratio between output and input power

$$
\varepsilon=\frac{P_{\text {out }}}{P_{\text {in }}} \text {. }
$$

The input power $P_{i n}=\langle G(t) v\rangle$ is supplied to the system by all external forces $G(t)$, i.e. $G(t)=a \cos (\omega t)+F$ or $G(t)=a \cos (\omega t)+\eta(t)$. To obtain its magnitude let us recast Eqs. (1) into the following form:

$$
\begin{aligned}
& d x=v d t, \\
& d v=-\left(\gamma v+V^{\prime}(x)-G(t)\right) d t+\sqrt{2 \gamma D_{T}} d W(t),
\end{aligned}
$$

where $W(t)$ is the Wiener process characterized by its two first moments $\langle W(t)\rangle=0$ and $\left\langle W^{2}(t)\right\rangle=t$. We now calculate the

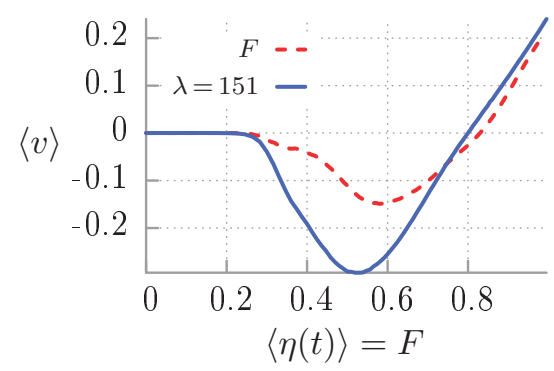

Fig. 1. The asymptotic long time stationary averaged velocity $\langle v\rangle$ of the studied Brownian particle is presented versus the magnitude of the constant $F$ or random $\langle\eta(t)\rangle$ bias in the ANM regime. Parameters are: $a=8.95, \omega=$ $3.77, \gamma=1.546, D_{T}=0.001, \lambda=151$.

differential of the dimensionless kinetic energy $v^{2} / 2$. In order to achieve this, we first use Ito's differential calculus getting

$$
\begin{aligned}
d\left(v^{2} / 2\right)= & -\left(\gamma v^{2}+v V^{\prime}(x)-G(t) v-\gamma D_{T}\right) d t \\
& +\sqrt{2 \gamma D_{T}} v d W(t) .
\end{aligned}
$$

Next, we perform the ensemble average for the rate of change of the kinetic energy

$$
\begin{aligned}
\frac{d}{d t} \mathbb{E}\left[v^{2} / 2\right]= & -\left(\gamma \mathbb{E}\left[v^{2}\right]+\mathbb{E}\left[v V^{\prime}(x)\right]\right. \\
& \left.-\mathbb{E}[G(t) v]-\gamma D_{T}\right),
\end{aligned}
$$

where for the part containing the Wiener process we exploited the Ito martingale property. Next, according to the formula (3), we average over the temporal period of the external harmonic force. Due to the periodicity of the asymptotic long time probability density [16] two terms are zero and we finally obtain

$$
\begin{array}{r}
P_{i n}=\gamma\left[\left\langle v^{2}\right\rangle-D_{T}\right]=\gamma\left[\left\langle v^{2}\right\rangle-\left\langle v^{2}\right\rangle_{e q}\right] \\
=\gamma\left[\langle v\rangle^{2}+\sigma_{v}^{2}-\left\langle v^{2}\right\rangle_{e q}\right],
\end{array}
$$

where, according to the equipartition theorem, in the thermodynamical equilibrium $\left\langle v^{2}\right\rangle_{e q}=D_{T}$. Note that the input energy depends not only on the force $G(t)$ but also (via $\left\langle v^{2}\right\rangle$ ) on all other parameters of the system.

Depending on the specific choice of the numerator $P_{\text {out }}$ in (5) different definitions of the Brownian motor efficiency characterize various aspects of energetics of the system. In particular, if the particle is working against a constant load $F$ then efficiency of energy conversion [17] can be defined as

$$
\varepsilon_{e}=\frac{|F\langle v\rangle|}{P_{i n}}=\frac{|F\langle v\rangle|}{\gamma\left(\left\langle v^{2}\right\rangle-D_{T}\right)} .
$$

Another possibility is to choose the friction force $F_{v}=\gamma v$ yielding the well known Stokes efficiency [18]

$$
\varepsilon_{s}=\frac{F_{v}\langle v\rangle}{P_{\text {in }}}=\frac{\gamma\langle v\rangle\langle v\rangle}{\gamma\left(\left\langle v^{2}\right\rangle-D_{T}\right)}=\frac{\langle v\rangle^{2}}{\left\langle v^{2}\right\rangle-D_{T}} .
$$

However, one should be aware of the fact that this is not exactly output power coming from work against the friction force as it should be proportional to $\left\langle v^{2}\right\rangle$ rather than $\langle v\rangle^{2}$ (see the discussion in [19]). Finally when we consider the kinetic 

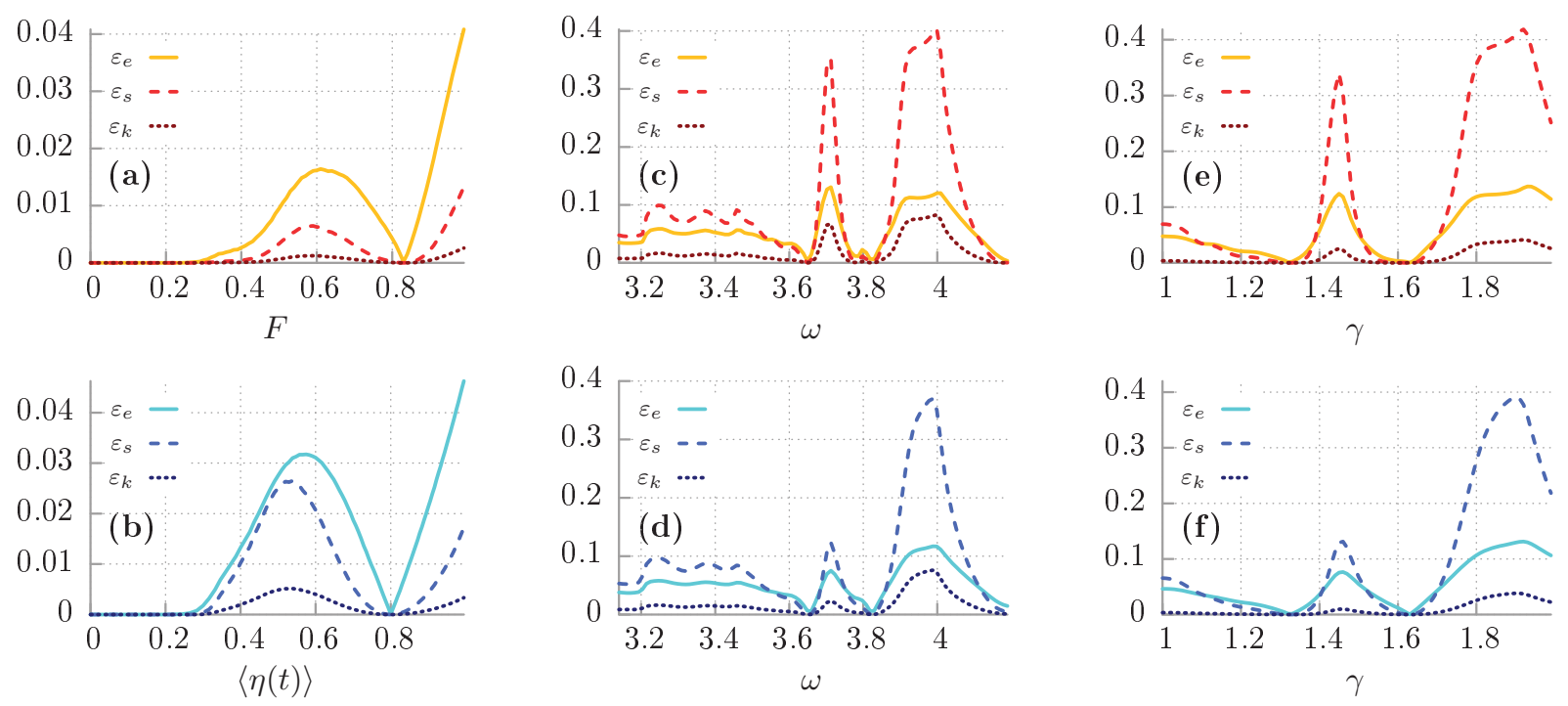

Fig. 2. The efficiency of energy conversion $\varepsilon_{e}$, the Stokes efficiency $\varepsilon_{s}$ and finally the kinetic efficiency $\varepsilon_{k}$ are presented as a function of the constant $F$ or stochastic $\langle\eta(t)\rangle$ bias (left panels), the angular frequency of the external harmonic driving $\omega$ (middle panels) and the friction coefficient $\gamma$ (right panels). Upper figures correspond to the scenario when the Brownian particle is subjected to the static bias $F$ whereas the bottom ones represent the case of the stochastic perturbation of equal mean value $\langle\eta(t)\rangle=F$. Other parameters are the same as in Fig. 1. Additionally, in panels (c)-(f) the bias has fixed constant value $\langle\eta(t)\rangle=F=0.58$.

energy of the Brownian particle per period of the external harmonic driving we get the so called kinetic efficiency

$$
\varepsilon_{k}=\frac{\left(\langle v\rangle^{2} / 2\right)(\omega / 2 \pi)}{\gamma\left(\left\langle v^{2}\right\rangle-D_{T}\right)}=\frac{\omega\langle v\rangle^{2}}{4 \pi \gamma\left(\left\langle v^{2}\right\rangle-D_{T}\right)} .
$$

Physical intuition tells us that large fluctuations $\sigma_{v}$ of the velocity reduce efficiency. In turn, when velocity fluctuations are small efficiency should be greater. All definitions of the motor efficiency presented above are consistent with this premonition. Put differently, transport is optimized in regimes which maximize the directed velocity and minimize its fluctuations.

The deterministic dynamics, i.e. when $D_{T}=D_{P}=0$ in (1) is extremely rich, complex and complicated [20], [21]. Depending on the parameter values, locked and running solutions can be observed. Moreover, dynamics involve periodic, subharmonic, quasiperiodic and chaotic motion. Since the FokkerPlanck-Kolmogorov-Feller master equation [15] corresponding to the white noise driven Langevin equations (1a) or (1b) surely cannot be solved analytically, we performed extensive numerical simulations. The specific details of the employed numerical code can be found in [22]. Here, we only mention that all numerical simulations were done by use of a CUDA environment which is implemented on a modern desktop GPU. This scheme allowed for a speed-up of a factor of the order $10^{3}$ as compared to a present-day CPU method [22], [23]. Our so obtained main results are presented next.

Due to high multidimensionality of the parameter space $\left\{\gamma, a, \omega, D_{T}, F, \lambda, D_{P}\right\}$ it is impossible to probe the full space numerically even for the powerful modern processing units. We therefore restrict our analysis to a particular, however interesting regime exhibiting the anomalous transport behavior in form of the ANM. We start with the asymptotic average velocity $\langle v\rangle$. This quantity is typically reduced when the constant deterministic force $F$ is replaced by the biased random perturbation $\eta(t)$, see Fig. 1. However, there are also regimes in the parameters space where $\eta(t)$ is more effective. The characteristic feature is emergence of the interval where for sufficiently large $\langle\eta(t)\rangle>0$ the random force can induce negative average velocity $\langle v\rangle<0$. Moreover, there exists an optimal value for the bias $\langle\eta(t)\rangle \approx 0.58$ at which the average velocity takes its minimal value. The most interesting is the fact that in the case of the stochastic force $\eta(t)$ the absolute value of $\langle v\rangle$ is nearly two times greater than in the corresponding deterministic case $F$.

Let us now analyze the Brownian motor efficiency in this particular regime exhibiting very intriguing anomalous transport features. The corresponding plots are depicted in Fig. 2. Panel (a) and (b) presents various previously introduced measures of Brownian motor effectiveness, i.e. the energy conversion $\varepsilon_{e}$, Stokes $\varepsilon_{s}$ and kinetic $\varepsilon_{k}$ efficiency as a function of the static deterministic $F$ and random $\langle\eta(t)\rangle$ bias, respectively. It follows from the definition that the energy conversion efficiency $\varepsilon_{e}$ approaches zero when the forces $\langle\eta(t)\rangle=F \rightarrow 0$. This is also the case in two other effectiveness quantifiers as they are both proportional to square of the asymptotic longtime stationary velocity $\langle v\rangle^{2}$ which is negligibly small for vanishing the symmetry breaking force of either deterministic $F$ or stochastic $\eta(t)$ nature. The reader can observe very congruent functional dependence of all types of efficiency. However, the energy conversion efficiency $\varepsilon_{e}$ is the largest of them, whereas the kinetic efficiency $\varepsilon_{k}$ is the smallest. The locally maximal efficiency of energy conversion $\varepsilon_{e}=0.0164$ for $F=0.61$ and $\varepsilon_{e}=0.0317$ for $\langle\eta(t)\rangle=0.57$, the Stokes efficiency $\varepsilon_{s}=0.0065$ for $F=0.59$ and $\varepsilon_{s}=0.0265$ for $\langle\eta(t)\rangle=0.54$, the kinetic efficiency $\varepsilon_{k}=0.0013$ for $F=0.59$ and $\varepsilon_{k}=0.005$ for $\langle\eta(t)\rangle=0.54$. The most important remark is the occurrence of an optimal value of force $\langle\eta(t)\rangle=F$ regardless of the definition of the efficiency. In particular, by use of the biased random force, Stokes and kinetic efficiencies 
grow by a factor of 4 and efficiency of energy conversion grows by the factor 2 over the value obtained with a deterministic force, compare the upper and bottom panels. This effect is directly related to the property that the stochastic force enhances the absolute value of velocity but also minimize its fluctuations [7].

Panel (c) and (d) depict the same efficiency measures but as a functions of the angular frequency $\omega$ of the external harmonic driving $a \cos (\omega t)$. Again, the dependence is similar regardless of the type of efficiency. However, now it is much more complicated being rather nonlinear and nonmonotonic curve. Here the Stokes efficiency is the largest of them. Notably, the kinetic one depends explicitly on the frequency of the driving $\omega$ and equals zero under its absence. One can observe that the efficiencies vanish for the sufficiently large angular frequency $\omega$. It is due to the fact that very fast oscillations of the harmonic driving significantly hamper the directed transport and consequently lower its efficiency. Not unexpectedly, there are intervals of $\omega$ for which the transport induced by the static bias $F$ is more effective than one caused by the random perturbation $\eta(t)$. However, there are also values of the angular frequency when the situation is completely opposite. The last two panels ((e) and (f)) shows various types of Brownian motor efficiency versus the friction coefficient $\gamma$. The Stokes efficiency possesses the largest efficiency. Similarly to the previous two panels the functional dependence behaves in nonmonotonic manner. Generally the efficiency of the directed transport process induced by the static bias $F$ or random forcing $\eta(t)$ is comparable. However, there exist windows of the friction coefficient $\gamma$ for which either the first or the second one is more efficient.

\section{SUMMARY}

We have studied in detail two scenarios of directed motion of the inertial Brownian motors: one driven by the deterministic force $F$ and the other propelled by biased non-equilibrium noise $\eta(t)$. In particular, we find domains in the parameters space such that when $F$ is replaced by $\eta(t)$ of equal average bias, the motor velocity is several times larger and its efficiency becomes considerably enhanced. Specific results are detailed for generalized white Poissonian noise and exemplified in the very intriguing regime of anomalous response in the form of an absolute negative mobility. The main conclusion, remain valid, however, as well for other models of random perturbations and also for the tailored transport regime showing a normal response (not depicted). Thus, the idea that random biased forces can be beneficial over deterministic biasing carries potential for practical realization in physics of Brownian motors. Moreover, the proposed mechanism of a nonequilibrium noise enhanced efficiency may explain exotic transport phenomena not only in physical but also in biological settings. It can be implemented in enhancing the working efficiency of synthetic molecular motors, for example, in a setup consisting of a the resistively and capacitively shunted Josephson junction device operating in corresponding experimentally accessible regimes.

\section{ACKNOWLEDGEMENT}

The work has been supported in part by the NCN grant DEC-2013/09/B/ST3/01659 (JS, JŁ) and the German Excellence Initiative "Nanosystems Initiative Munich"( $\mathrm{PH})$.

\section{REFERENCES}

[1] L. Gammaitoni, P. Hänggi, P. Jung and F. Marchesoni, "Stochastic Resonance", Rev. Mod. Phys., vol. 70, p. 223, 1998.

[2] P. Reimann, "Brownian motors: noisy transport far from equilibrium", Phys. Rep., vol. 361, p. 57, 2002.

[3] P. Hänggi and F. Marchesoni, "Artificial Brownian motors: Controlling transport on the nanoscale", Rev. Mod. Phys., vol. 81, p. 387, 2009.

[4] E. R. Kay, D. A. Leigh and F. Zerbetto, "Synthetic molecular motors and mechanical machines", Angewandte Chemie International Edition, vol. 46, p. 72, 2007.

[5] P. C. Bressloff and J. M. Newby, "Stochastic models of intracelluar transport", Rev. Mod. Phys., vol 85, p. 135, 2013.

[6] P. Reimann, C. Van den Broeck, H. Linke, P. Hänggi, J. M. Rubi and A. Pérez-Madrid, "Giant acceleration of free diffusion by use of tilted periodic potentials", Phys. Rev. Lett., vol. 87, p. 010602, 2001.

[7] J. Spiechowicz, P. Hänggi and J. Łuczka, "Brownian motors in the microscale domain: Enhancement of efficiency by noise", Phys. Rev. E, vol. 90, p. 032104, 2014.

[8] M. Kostur, Ł. Machura, P. Hänggi, J. Łuczka, and P. Talkner, ’Forcing inertial Brownian motors: efficiency and negative differential mobility", Physica A, vol. 371, p. 20, 2006.

[9] Ł. Machura, M. Kostur, P. Talkner, J. Łuczka and P. Hänggi, "Absolute negative mobility induced by thermal equilibrium fluctuations", Phys. Rev. Lett., vol. 98, p. 40601, 2007.

[10] D. Speer, R. Eichhorn and P. Reimann, "Brownian motion: Anomalous response due to noisy chaos", Europhys. Lett., vol. 79, p. 10005, 2007.

[11] J. Nagel, D. Speer, T. Gaber, A. Sterck, R. Eichhorn, P. Reimann, K. Ilin, M. Siegel, D. Koelle, and R. Kleiner, "Observation of negative absolute resistance in a Josephson junction", Phys. Rev. Lett., vol. 100, p. 217001, 2008.

[12] M. Kostur, Ł. Machura, P. Talkner, P. Hänggi and J. Łuczka, "Anomalous transport in biased ac-driven Josephson junctions: Negative conductances", Phys. Rev. B, vol. 77, p. 104509, 2008.

[13] J. Spiechowicz, P. Hänggi and J. Łuczka, "Josephson junction ratchet: The impact of finite capacitances", Phys. Rev. B, vol. 90, p. 054520 , 2014.

[14] J. Spiechowicz, J. Łuczka and P. Hänggi, ”Absolute negative mobility induced by white Poissonian noise", J. Stat. Mech., p. P02044, 2013.

[15] P. Hänggi, "Langevin description of Markovian integro-differential master equations", Z. Phys. B, vol. 36, no. 3, p. 271, 1980.

[16] P. Jung, "Periodically driven stochastic systems", Phys. Rep., vol. 234, p. $175,1993$.

[17] Ł. Machura, M. Kostur, P. Talkner, P. Hänggi, and J. Łuczka, ”Negative conductances of Josephson junctions: Voltage fluctuations and energetics", Physica E, vol. 42, p. 590, 2010.

[18] Ł. Machura, M. Kostur, P. Talkner, J. Łuczka, F. Marchesoni and P. Hänggi, "Brownian motors: Current fluctuations and rectification efficiency", Phys. Rev. E, vol. 70, p. 061105, 2004.

[19] J. Spiechowicz and J. Łuczka, "Efficiency of the SQUID ratchet driven by external current", New. J. Phys., vol. 17, p. 023054, 2015.

[20] P. Jung, J. G. Kissner, and P. Hänggi, "Regular and Chaotic Transport in Asymmetric Periodic Potentials: Inertia Ratchets", Phys. Rev. Lett. vol. 76, p. 3436, 1996.

[21] J. L. Mateos, "Chaotic transport and current reversal in deterministic ratchets", Phys. Rev. Lett., vol. 84, p. 258, 2000.

[22] J. Spiechowicz, M. Kostur and Ł. Machura, "GPU accelerated Monte Carlo simulation of Brownian motors dynamics with CUDA", in press in Comp. Phys. Commun., DOI:j.cpc.2015.01.021, arXiv:1409.4923, 2015.

[23] M. Januszewski and M. Kostur, "Accelerating numerical solution of stochastic differential equations with CUDA", Comp. Phys. Commun., vol. 181, p. 183, 2010. 